\title{
Contacto mapudungun-castellano: usos, transmisión y valoraciones en comunidades pewenche del Alto Biobío*
}

\author{
Mapudungun-spanish contact: uses, transmission and \\ valuations in pewenche del Alto Biobío communities
}

\author{
Marisol Henríquez Barahona** , Irina Mellado Ríos***, \\ Octavio Abello Segura ${ }^{* * * *}$ y Pamela Parizot Troncoso*****
}

\section{RESUMEN}

El artículo examina la situación del mapudungun en contacto con el castellano en comunidades pewenche del Alto Biobío. En específico, se describe el estatus de la lengua a través del análisis de su uso y transmisión intergeneracional, además de abordar las valoraciones que manifiestan los hablantes hacia ambas lenguas. La muestra estuvo conformada por el discurso de diez hablantes bilingües de dos comunidades del Valle del Queuco. Los datos fueron recogidos a través de entrevistas semiestructuradas que permitieron obtener información sociolingüística del hablante, su familia y la comunidad. En términos generales es posible señalar que en la zona existe un mantenimiento de la lengua bastante disímil que se evidencia en patrones de uso y transmisión heterogéneos. No obstante, las valoraciones hacia ambas lenguas son positivas.

\section{SUMMARY}

The article examines the circumstances of Mapudungun coming into contact with Spanish in Pewenche communities in the
Palabras

clave: español, mapudungun, transmisiónvaloraciones sociales, uso de la lengua.

Keywords: Spanish, Mapudungun,

\footnotetext{
* Los resultados de este trabajo están asociados al Proyecto Fondecyt de Iniciación 11150508 y a la Tesis para optar al Grado de Licenciado en Educación de los coautores. ** Chilena. Doctora en Lingüística de la Universidad de Concepción, investigadora de la Universidad Católica de la Santísima Concepción. Concepción, Chile, mhenriquez@ucsc.cl *** Chilena, Magíster en Lingüística de la Universidad de Chile. Universidad Católica de la Santísima Concepción. Concepción, Chile, irinamelladorios@gmail.com

**** Chileno. Licenciado en Educación de la Universidad Católica de la Santísima Concepción. Concepción, Chile, oabello@emlenguaje.ucsc.cl

***** Chilena, Licenciada en Educación de la Universidad Católica de la Santísima Concepción. Concepción, Chile,pparizot@emlenguaje.ucsc.cl
} 
Alto Biobío. Specifically, the status of the language is described through the analysis of its use and intergenerational transmission, in addition to addressing the assessments that speakers express towards both languages. The sample consisted of the speech of ten bilingual speakers from two communities in the Queuco Valley. The data was collected through semi-structured interviews that allowed researchers to obtain sociolinguistic information from the speaker, their family and community. In general terms, it can be said that there is a fairly dissimilar maintenance of the language in the area that is evidenced in heterogeneous patterns of use and transmission. However, the appreciation of both languages is positive. transmissionsocial evaluations, use of the language. 


\section{Introducción}

En los últimos años, una serie de investigaciones ha documentado y manifestado que existe un retroceso en el mantenimiento de la lengua mapuche, que se evidencia a partir del análisis de categorías sociolingüísticas tales como el número de hablantes, la transmisión intergeneracional y los niveles de competencia en la lengua (Alonqueo et al., 2017; Gundermann et al., 2009, 2011; Lagos, 2012; Wittig y Alonqueo, 2018; Zúñiga, 2007; Zuñiga y Olate, 2017).

Según algunos estudios, este escenario poco favorable se caracteriza por la imposición del castellano y la creciente pérdida de funcionalidad del mapudungun o chedungun ${ }^{1}$, lo que al parecer tendría directa relación con la reducción de espacios sociales donde la lengua originaria puede ser desplegada (Gundermann et al., 2009; Lagos, 2006; Wittig, 2009). No obstante, esta situación no es homogénea en los distintos territorios, pues existen algunas zonas, especialmente rurales, que presentan un panorama más alentador para la lengua.

Procesos históricos, sociales, económicos y culturales particulares de cada zona, han configurado este complejo panorama, el que podemos observar, por ejemplo, en la Región del Biobío. Por un lado, la zona lafkenche de la provincia de Arauco, escenario histórico de diversos conflictos territoriales y con un contacto fluido entre la sociedad mapuche y la sociedad nacional (Gundermann et al., 2008), se caracteriza por presentar bajos niveles de bilingüismo y donde el mapudungun ha quedado relegado a los dominios familiares íntimos, en los que participan principalmente los mayores; también a contextos más formales como ceremonias ancestrales (Henríquez, 2014). Por otro lado, y en contraste, la zona pewenche del Alto Biobío exhibe una situación más favorable para la lengua, puesto que sus usos se despliegan de manera transversal en contextos cotidianos, tradicionales y no tradicionales y, además, se observa una mayor transmisión de la lengua (Henríquez, 2014). No obstante, este panorama no es extensivo a todas las comunidades del territorio, pues se ha observado una diversidad en el comportamiento sociolingüístico de las familias, producto de

1 En este artículo utilizaremos indistintamente las denominaciones mapudungun y chedungun para referirnos a la lengua de los pewenches; pues de esta manera, los colaboradores que participaron de este estudio designan su lengua. 
diversos procesos de intervención y contacto, los que han impactado en las decisiones y prácticas lingüísticas de sus hablantes. Así, algunas comunidades pewenche presentan un mayor uso y mantenimiento de la lengua, mientras que otras experimentan una dinámica de cambio lingüístico que debilita su práctica y fortalece el uso de la lengua hegemónica (Dinamarca y Henríquez, 2019; Henríquez, 2014, 2015; Henríquez y Dinamarca, 2018; Lagos, 2017; Ojeda y Álvarez, 2014).

Este panorama diverso que los especialistas han descrito para la comuna de Alto Biobío, deja en evidencia la relevancia de no asumir que las comunidades que tienen proximidad geográfica poseen necesariamente una práctica sociolingüística similar u homogénea. Por lo mismo, creemos importante desarrollar estudios cualitativos focalizados, con la finalidad de ahondar en las situaciones bilingües particulares que viven las distintas comunidades del sector y, como pretendemos en este trabajo, avanzar en la configuración de un diagnóstico de la relación de las lenguas en contacto, a través de un estudio de caso en comunidades de habla específica, considerando dimensiones como el uso, la transmisión y las valoraciones.

\section{Contacto y valoraciones sociolingüísticas}

El español, como lengua hegemónica, se posiciona como uno de los principales instrumentos del modelo sociopolítico y económico de occidente en América Latina y como parte del proyecto de conquista y colonización ha construido un enorme muro que dificulta cualquier existencia cultural y lingüística alterna. En el ámbito sociolingüístico, la relación de dominancia entre el castellano y las lenguas originarias se traduce en la minorización de estas últimas, que progresivamente pierden espacios sociales y lingüísticos frente a la lengua española, que se posiciona como la lengua del poder oficial, es decir, un instrumento de administración y de subordinación ${ }^{2}$ (Godenzzi, 2017). En esta dinámica de contacto y subordinación, el castellano empieza a ser integrado por la comunidad de habla minoritaria y se produce lo que Godenzzi (2007) denomina un proceso de interactividad lingüisti-

2 Según Soto (2014) pensar en el mapuzugun es pensar esta lengua indígena en relación con la lengua nacional, en este caso el castellano; es pensar en la cuestión del poder, del poder decir, del poder — que se tiene o no se tiene- para decir (se). 
$c a$, que se entiende como la relación antagónica existente entre dos o más tradiciones lingüísticas, en determinados ámbitos sociales, que se nutren en una doble dirección. Esta asimetría significa el inicio de un escenario de dominancia sociolingüística.

En Chile, la castellanización y educación forzada de la población mapuche, en el marco de procesos sociopolíticos de colonización y construcción de un sistema nacional, ha provocado el innegable retroceso de la lengua originaria, hecho que se evidencia en la disminución del bilingüismo, la pérdida de espacios de uso tradicionales y el avance del castellano en ámbitos que tradicionalmente le pertenecían al mapudungun (Gianelli, 2005; Henríquez, 2015; Lagos, 2017; Olate et al., 2013). Este proceso de incorporación del castellano ha sido gradual, y viene potenciado por la presión socioeconómica y comunicativa que ejerce la sociedad hegemónica sobre los hablantes de las comunidades ${ }^{3}$.

En el marco de esta dinámica de dominancia e intervención, el mantenimiento del mapudungun en Chile podría interpretarse como el resultado de diversos estilos de resistencia cultural por parte del grupo dominado ante los avances constantes y variados que ejerce la dominación de la cultura occidental hispanohablante sobre el pueblo mapuche (Durán et al., 2007). Este acto de resistencia se observa cuando los hablantes de comunidades minorizadas insisten en hablar su lengua pese a todos los actos coercitivos que despliega el Estado y la sociedad dominante para que la abandonen ${ }^{4}$ (Calvet, 2005).

Ahora bien, la percepción de los hablantes de esta minorización o conflicto lingüístico no contribuye a fomentar la mantención de la lengua ni la construcción de valoraciones positivas, lo que tiene consecuencias directas en el comportamiento sociolingüístico de las comunidades de habla y, en definitiva, en el desarrollo de procesos de revitalización lingüística. Según Crystal (2001), "las lenguas decaen cuando faltan estas actitudes positivas y, en muchos casos faltan, porque a menudo, por motivos políticos se enfrentan a un entorno hostil" (p. 97).

3 De acuerdo con Rojas (2013), para construir la identidad chilena en el siglo XIX, la elite hispanohablante desarrolló una ideología lingüística que favorecía al español, a través de procesos de iconización y ocultamiento.

4 Según Soto, respecto de la acción del Estado se podría hablar "de más de un siglo de apagamiento e interdicción de las lenguas y memorias de las denominadas 'minorías étnicas' en Chile" (2014). 
Las valoraciones subjetivas y sociales que tienen los hablantes hacia las lenguas son uno de los factores sociolingüísticos de relevancia en el estudio de las lenguas en interacción, pues afectan y direccionan las decisiones lingüísticas de los miembros de la comunidad de habla y, además, nos permiten conocer la situación de una lengua desde dentro de la comunidad. Eso sí, es relevante añadir que no solo son preponderantes las actitudes o valoraciones de las comunidades minoritarias, sino también aquellas que manifiestan los hablantes de la sociedad dominante o mayoritaria.

Efectivamente, las valoraciones o actitudes lingüísticas constituyen un aspecto importante de la compleja psicología social de las comunidades lingüísticas - especialmente de las bilingües-y corresponden a una manifestación de la actitud social del individuo. Están centradas y referidas tanto a la lengua como al uso que se hace de ella en contextos sociales concretos (Moreno Fernández, 2012). Estas valoraciones lingüísticas se pueden definir como entidades psicológicas complejas que conllevan conocimiento y sentimiento, además de comportamiento (Díaz Campos, 2014; Garret, 2010; Hernández Campoy, 2004); se perfilan como valoraciones positivas o negativas que tienen los hablantes hacia una lengua o variedad determinada (Blas Arroyo, 2008) y constituyen un conjunto de creencias socialmente compartidas (Moreno Fernández, 2012).

En otras palabras, se trata de una disposición a reaccionar de manera positiva o negativa hacia la forma de hablar de las personas, considerando la pronunciación, el uso de una estructura lingüística o la elección de una lengua en lugar de otra (Edward, 2009). En una situación de bilingüismo social y conflicto lingüístico - como el que se aborda en este estudio-, las valoraciones propician que algunas variedades o lenguas se confinen a los dominios menos formales y otras predominen en los de mayor prestigio y formalidad, lo que incluso puede llevar, si la actitud es negativa o desfavorable, al abandono y olvido de una lengua (Moreno Fernández, 2009).

Por último, en toda comunidad de habla, la lengua desempeña un papel importante en la configuración de los sentimientos de identidad etnolingüística de los hablantes, lo que se manifiesta en las valoraciones que expresan hacia sus lenguas. Aunque es necesario mencionar que la identificación de una comunidad con una lengua y su cultura y 
las valoraciones positivas no siempre son garantía de pervivencia de las lenguas, sin embargo no se puede negar que "al menos como punto de partida constituyen un pilar importante para su normalización social" (Blas Arroyo, 2008, p. 469). Esto quiere decir que, aunque los factores sociales sean favorables para que ocurra el desplazamiento lingüístico del grupo minoritario, las actitudes de los hablantes pueden generar excepciones incluso en los escenarios más desalentadores (Thomason, 2001). De ahí entonces la necesidad de indagar en estas valoraciones tanto por su impacto en las prácticas comunicativas, especialmente en el uso y transmisión de la lengua; como por la oportunidad de discernir respecto de la dinámica de funcionamiento de las lenguas en relación y los procesos de cambio que vive la lengua mapudungun (Olate y Henríquez, 2010; Henríquez y Dinamarca, 2018).

\section{La investigación}

El presente trabajo, en concordancia con el objeto de estudio, se adscribió al paradigma cualitativo y fue de tipo exploratorio. El método consistió un estudio de caso, ya que examinó las semejanzas y diferencias de casos particulares (Langley y Royer, 2006).

\section{Objetivo y participantes}

El objetivo que nos hemos propusimos fue describir la situación del chedungun en contacto con el español en las comunidades pewenche de Pitril y Butalelbun, a través del análisis de los usos, la transmisión intergeneracional y las valoraciones que manifiestan los hablantes hacia ambas lenguas. Esto nos permitió ahondar en los procesos de conservación y desplazamiento de la lengua y su relación con el castellano en un territorio específico.

Ambas zonas de estudio, Pitril y Butalelbun, se encuentran en la comuna de Alto Biobío. Pitril se emplaza a 11 kilómetros de la Villa Ralco. Su actividad económica está asociada a la ganadería, una incipiente industria turística y la agricultura a pequeña escala, debido a que la falta y deterioro del terreno limita los espacios en donde las familias pueden asentarse para cultivar pequeñas huertas y mantener los animales. La comunidad posee una posta de salud rural, una escuela básica adscrita al Programa de Educación Intercultural Bilingue (PEIB) y señal telefónica. Una característica importante de esta comunidad es 
que, al momento de realizar la recolección de datos, no existía la figura de una autoridad tradicional o un longko, ya que la organización de la comunidad giraba en torno al trabajo del presidente de la comunidad, quien obraba de la mano de la Corporación Nacional de Desarrollo Indígena, Conadi.

Por su parte, Butalelbun se encuentra a 62 kilómetros de Ralco y a 10 kilómetros de Copahue, Argentina. Es la comunidad pewenche más apartada del Valle del Queuco. Su principal actividad económica es la agricultura y ganadería de subsistencia. Posee un retén de carabineros, una posta de salud primaria y una escuela adscrita al PEIB. Al momento del trabajo de campo, se estaban haciendo gestiones para la implementación de la señal telefónica. A diferencia de Pitril, en esta comunidad sí existía un longko, escogido por los miembros de la comunidad.

Se trabajó con una muestra intencionada (Hernández-Campoy y Almeida, 2005) de 10 hablantes bilingües, cinco pertenecientes a la comunidad de Pitril y cinco a la de Butalelbun. Todos los participantes han vivido durante toda su vida en las comunidades y son mayores de 18 años.

\section{Procedimientos}

Para la recolección de datos se aplicó una entrevista sociolingüística, que se dividió en tres dimensiones: usos de las lenguas, transmisión del chedungun y valoraciones sociales; además, a modo de complemento, se empleó el mecanismo de la observación in situ que realizaron los investigadores durante el trabajo de campo. Cada entrevista contó con el consentimiento oral de los entrevistados y se registró mediante el método de grabación de audio. Ambas estrategias tuvieron por objetivo recoger la mayor cantidad de información sociolingüística respecto de la situación del chedungun y su relación con el castellano dentro de la comunidad.

\section{Resultados}

\section{Uso de las lenguas}

En la presente dimensión se describen los ámbitos o espacios comunicativos en que los hablantes hacen uso de su lengua, los que para 
efectos del presente estudio se clasifican en dos tipos: (1) intra y extracomunitarios y (2) tradicionales y no tradicionales. Los espacios intra y extracomunitarios corresponden a una abstracción teórica para discernir entre aquellos que se sitúan dentro o fuera de la comunidad. Mientras que los tradicionales y no tradicionales se refieren a los contextos originalmente pertenecientes a la cultura pewenche, versus los propios de la cultura occidental hegemónica.

Tanto en Butalelbun como en Pitril se utiliza el chedungun en diferentes espacios socioculturales. No obstante, se observa un importante contraste entre ambas comunidades. Por un lado, en Pitril, la lengua originaria se emplea principalmente en contextos intracomunitarios tradicionales, como en el hogar, en los caminos, en las ceremonias ancestrales, etc., siempre y cuando participen adultos o mayores en la conversación, puesto que en esta comunidad los jóvenes y niños que hablan la lengua pewenche son escasos. Aunque todos los entrevistados, hablantes de mapudungun, señalaron que en el caso de los niños y jóvenes la situación es diferente:

acá (Pitril) uno se acostumbra, que aquí todos hablan el español, entonces ... es como una costumbre (L. P., fem., Pitril).

Lo observado aquí coincide con lo señalado por Lagos (2017) en relación con el desplazamiento funcional del mapudungun al interior del núcleo familiar. El uso de la lengua se limitaría a la conversación entre adultos y mayores, producto de la interrupción de la transmisión a nuevas generaciones, "derivado de expectativas sociales asociadas al español y la experiencia pasada de discriminación" (p. 69).

Según los colaboradores, en la comunidad existen hablantes de chedungun con diversos grados de competencia; sin embargo, no todos hacen uso del idioma con la misma frecuencia en todos los espacios de interacción. Su empleo es disímil y en permanente interactividad con el castellano (Godenzzi, 2007). Como ejemplo de este proceso, observamos los fenómenos de mezcla y alternancia lingüística que caracterizan el uso de la lengua en Pitril, situación que sería consecuencia de la prolongación de las relaciones coloniales al interior de las comunidades mapuche (Ojeda y Álvarez, 2014). No obstante, coincidimos con estos autores en que este fenómeno sociolingüístico también constituye un símbolo de resistencia frente a la 
aculturación impuesta por el mundo occidental. Resulta interesante que los propios hablantes sean conscientes de estos procesos y del dinamismo que atraviesa su lengua patrimonial:

en la familia ya no se está practicando [el chedungun], no se está dando una preferencia al chedungun. $O$ sea, si yo me junto con un peñi de mi edad, ¡claro! vamos a conversar ... harto rato, de todo. Pero ya cuando está en la casa, con la familia ... está el castellano primero, el castellano ... aunque uno no lo quiera, está conversando en chedungun y de repente... el castellano se mete al tiro. Estamos muy acostumbrados a esa forma de hablar, a esa forma de entender (L. Ll., masc., Pitril).

Respecto del castellano, en esta comunidad se emplea en la mayoría de los contextos comunicativos inclusive, como lo señala este colaborador, al interior del hogar, sobre todo en conversaciones en las que participan niños y jóvenes. Los procesos históricos de discriminación y castigo por hacer uso del chedungun favorecieron la incorporación gradual del castellano como estrategia comunicativa de sobrevivencia. Asimismo, la influencia de la escuela, sinónimo de progreso y estatus social, propicia el uso de la lengua oficial en desmedro del idioma originario. Lo mismo ocurre con iniciativas económicas relacionadas con el turismo - comunes en esta comunidad-y que generan instancias comunicativas predominantemente en castellano.

Otro espacio comunicativo que favorece el empleo de esta lengua es el de las ceremonias evangélicas. En Pitril se observa un gran número de templos evangélicos a los que asiste la mayoría de las familias de la localidad y donde se habla principalmente en castellano. Algunos entrevistados reconocen el impacto de esta práctica religiosa en la participación de espacios sociocomunicativos religiosos ancestrales como la ceremonia del ngillatun:

uno es libre de tener cualquier religión, pero afecta mucho en el tema en que el peñi siendo evangélico, nos comentaba, eh ... acá no pueden participar en el nguillatun, porque según ellos la religión lo prohíbe (L. Ll., masc., Pitril).

En esta comunidad, al igual como se ha descrito para otros territorios, la figura de la iglesia se relaciona con la pérdida de la lengua y de las creencias mapuche (Wittig y Hernández, 2017). 
En Butalelbun, por su parte, la lengua se emplea en toda interacción comunicativa, tanto tradicional como no tradicional y sin importar la edad de los interlocutores.

(En Butalelbun) en todas partes (se habla chedungun), porque uno lo hace porque es así, uno nació con el chedungun, uno nunca tiene que perderlo, ... hay gente que va a la ciudad y no está ni ahí con hablar el chedungun porque yo creo que se avergüenzan o porque les hacen bullying, pero la gente de Butalelbun a donde sea que vaya hablan en chedungun, porque se sienten orgullosos de ser pewenche (M. M., masc., Butalelbun).

Para los hablantes de Butalelbun, el chedungun trasciende los espacios intracomunitarios. El orgullo e identificación con su cultura y lengua les permite utilizarla incluso en la ciudad, lugar donde los miembros de otras comunidades como Pitril, optan por el castellano para evitar instancias de discriminación y estigmatización de parte de los chilenos. Según declaran algunos colaboradores de Butalelbun, no hay límites geográficos a la hora de utilizar la lengua originaria.

Yo en todas partes hablo chedungun, hasta fui a Copiapó y ahí hablé en mi idioma y los demás peñis me decían “ipor qué hablai chedungun? ¿por qué no dejai de hablar?" entonces les dije "peñi, yo nunca voy a olvidar mi idioma, y voy a hablarlo siempre, aunque tú no creas, o no quieras, tú no me puedes discriminar, tú estás discriminando a tu propia sangre" (F. M., masc., Butalelbun).

Lo narrado por este hablante se puede interpretar como una manifestación de resistencia lingüística y cultural, característica de algunas comunidades, donde la lengua, además de ser un símbolo de identidad y de diferenciación social se constituye en un refugio; lo que Calvet (2005) denomina un "espacio privilegiado de la autenticidad negada como último refugio contra la alienación colonial” (p. 182).

En Butalelbun, el uso del castellano está restringido a las interacciones con colonos, como por ejemplo con profesores no hablantes, con personal de la posta y autoridades comunales, con la policía y también en algunos eventos religiosos de la iglesia evangélica liderados por un pastor que no es de la comunidad. Si bien el panorama en Butalelbun es auspicioso, la incorporación de estos espacios no tradicionales in- 
traétnicos podría implicar la retracción y marginación de la lengua originaria dentro de los mismos (Olate, et al., 2017).

\section{Transmisión intergeneracional del chedungun}

De esta dimensión se desprenden dos tipos de parámetros que emergen de los discursos de los entrevistados acerca de la transmisión de la lengua. Por un lado, la transmisión tradicional que recae en la familia y en las instituciones ancestrales y, por otro, aquella no tradicional ejercida principalmente por la escuela.

En la comunidad de Pitril, los entrevistados reconocen el valor y la importancia de la transmisión intergeneracional para la adquisición y conservación de la lengua. No obstante, la mayoría señaló que existen dificultades a la hora de transmitirla en sus propios núcleos familiares, donde la práctica el chedungun como primera lengua es escasa. Este fenómeno ha sido descrito por Gundermann (2014), quien señala que existe una tensión o ambivalencia entre el sistema de valoraciones y la práctica o transmisión de la lengua, lo que obedece a múltiples factores, entre los cuales destaca la necesidad de aprender y transmitir el castellano como una estrategia de sobrevivencia y movilidad social. Esta es una de las causas por las que ha disminuido la práctica del chedungun.

Además, que [el chedungun] no se practica, en la familia ya no se está practicando, no se está dando una preferencia al chedungun (L. Ll., masc., Pitril).

La mayoría de los colaboradores reconoce que el circuito de transmisión padre-hijo se ha debilitado o interrumpido en la comunidad, no obstante, se destaca el rol de los abuelos en la transmisión de la lengua.

No aprendí a hablar chedungun desde chico, por el prejuicio de mis padres, de que si yo aprendía a hablar o hablaba chedungun, no iba a aprender a leer. Ese es un tema que tocó harto en mi familia (Á. Ll., masc., Pitril).

El hogar representa uno de los espacios de transmisión lingüística y cultural más relevantes dentro de la comunidad. Por lo tanto, la interrupción de la enseñanza en el núcleo familiar (y otros dominios íntimos y tradicionales) trae consigo la disminución de hablantes (Henríquez, 2015; Lagos, 2017). 
Según los colaboradores, el sentimiento de vergüenza y miedo de algunos integrantes de la comunidad es uno de los principales obstáculos para usar y transmitir la lengua.

Los de Pitril algunos hablan, pero no todos, porque dicen que tienen vergüenza porque aún los chilenos creo que se ríen de uno, porque dicen que a lo mejor lo están retando, lo están tratando mal. Entonces prefieren hablar en español pa' que entiendan (R. V., masc., Pitril).

Estos sentimientos son consecuencia de las experiencias de discriminación que han vivido y aún viven las familias del sector. Se suma a esto la percepción negativa del bilingüismo como un obstáculo que dificulta el aprendizaje del castellano y, por ende, la promoción escolar. Lamentablemente esta creencia no es exclusiva de las familias, sino que está presente también en las escuelas. En general, se trata de una creencia muy extendida, especialmente en ámbitos educacionales, donde el bilingüismo no es bien visto, puesto que se asocia con desventajas cognitivas y educativas, sobre todo en niños de edad escolar (Montrul, 2013). Una labor pendiente de las instituciones formadoras es la modificación de esta percepción negativa de la lengua originaria como barrera para la comunicación intercultural y para la integración.

Contrariamente al escenario anterior, la transmisión y uso de la lengua en la comunidad de Butalelbun adquiere un valor cultural preponderante según relatan los entrevistados. La lengua, para esta comunidad, es un símbolo de orgullo y lealtad lingüístico-cultural y genera sentimientos de compromiso y responsabilidad hacia su conservación (Henríquez, 2014; Henríquez y Dinamarca, 2018). En este sentido, prácticamente todos los miembros del grupo familiar son agentes naturales de transmisión.

Los testimonios personales adquieren un peso esencial, lo cual reafirma lo expuesto por Ghio y Fernández (2008) en cuanto a que el entorno social y cultural en el que los hablantes adquieren el lenguaje no puede separarse de la construcción de realidad que crean los mismos.

Yo soy nacido y criado en chedungun $¡$ Yo no puedo perder mi lengua! No puedo, porque yo, sea como sea, yo tengo que hablar, porque tengo a quien enseñarle también, a mis hijos. Y yo quiero que 
ellos cuando sean grandes sepan lo que es el chedungun de nosotros, que no se pierda eso (M. V., fem., Butalelbun).

La conservación de la lengua y su constante transmisión en esta comunidad, trae consigo valoraciones positivas, no solo para sus miembros, sino también para otras comunidades del valle, como Pitril, donde se destaca y se reconoce a Butalelbun como un referente lingüístico, comunidad portadora de una variante normativa y de prestigio altamente valorada.

en mi opinión, el primero [el primer lugar], yo creo que es Butalelbun-Trapa. Ellos son súper... ellos hablan netamente el chedungun. Porque lo he visto en niñitos chicos poh, o sea de un año, dos años, hablan... son hablantes fluidos (L. P., fem., Pitril).

Los procesos de sustitución de la lengua que se observan en otras comunidades del territorio pewenche no se han instalado aún en la comunidad de Butalelbun, constituyéndose en una zona de alta vitalidad lingüística.

\section{El castellano, la lengua del trabajo}

De acuerdo con la información recogida en el trabajo de campo, tanto mediante las entrevistas como la observación in situ, el castellano se percibe en ambas comunidades como una lengua prioritariamente práctica, una herramienta y estrategia funcional que permite la incorporación y desenvolvimiento en la sociedad dominante. Como lengua oficial y del Estado se representa, entonces, como el principal motor de ascenso y movilidad social tanto en Pitril como en Butalelbun.

Para nosotros el español nos sirve para educar a nuestros hijos, ya que como dije anteriormente, el gobierno nunca va a hacer un texto en chedungun (M.M., masc., Butalelbun).

Porque a los viejos no les gusta enseñar [chedungun] porque dicen que cuando entran a la escuela no saben hablar [castellano], no entienden nada (R.V., masc., Pitril).

La percepción del castellano es la de una herramienta que facilita el desarrollo laboral incluso dentro de la comunidad. Su manejo y uso se relaciona con el mejoramiento del sustento económico familiar, pues facilita la apertura de nuevas posibilidades laborales a través de la formulación de proyectos, la vinculación con organizaciones guberna- 
mentales y no gubernamentales, así como el desarrollo de iniciativas derivadas de la industria. Esta presión socioeconómica de la comunidad lingüística hegemónica se refleja en la respuesta que entrega uno de los colaboradores ante la consulta acerca de la importancia o necesidad de saber español.

Estamos como obligados a eso [saber castellano], por todas estas ONG que entran a las comunidades ... que si no sabemos castellano, no podemos conseguir beneficios (L.Ll., masc., Pitril).

si usted no sabe hablar en español ...si no tengo mi curso, no tengo trabajo (H.M., masc., Butalelbun).

La presión socioeconómica de la comunidad lingüística dominante motiva el uso y valoración hacia el castellano. En conclusión, en ambas comunidades se evidencia el rol funcional de esta lengua, que domina los espacios educativos y permite mejoras sociales. Aunque esta representación se observa en mayor medida en los discursos de los hablantes de Pitril.

\section{Valoraciones hacia el chedungun}

A pesar del avance e imposición del castellano, los colaboradores de ambas comunidades se representan como integrantes de un circuito tradicional de producción y reproducción de la lengua (Lagos, 2017), en el que esta no solo es portadora de un valor comunicativo, sino de un valor cultural e identitario.

nosotros tenemos nuestra creencia, y nuestra creencia no está escrita en ninguna parte. O sea, el kimün, el rakiduam ... pensar, conocimiento, que vienen de cientos de años, de los bisabuelos, que hoy en día no lo tiene nadie, pero aquí cada persona o comunidad sí lo tiene. Entonces, por eso le decía que es un don de dios [hablar chedungun], porque no está escrito en los libros ... Entonces por eso es muy bueno esto de cultivar el chedungun, ojalá mantenerlo (L.Ll., masc., Pitril).

Se trata de un ejercicio de resistencia y pertenencia a un pueblo originario, donde el lenguaje juega un rol trascendental. Los colaboradores se reconocen como depositarios de toda una tradición ancestral, donde el chedungun representa la pertenencia, permanencia y perpetuación de ese saber. 
Por consiguiente, que exista una alta valoración hacia el chedungun permite que ese sentimiento de reivindicación identitaria de parte de los hablantes, especialmente presente en Butalelbun, genere esfuerzos por resistir a la intromisión de elementos foráneos en la estructura de su lengua o a fenómenos de mezcla o alternancia de códigos. Esto se observa en la respuesta del siguiente colaborador de Butalelbun cuando se le consulta acerca de esta situación.

acá si nosotros tenemos una conversación todo se habla en chedungun y itodo! tiene que ser en chedungun, no se mezcla (M. V., fem., Butalelbun).

En Pitril, en cambio, no se observa el mismo nivel de rechazo a la presencia de elementos foráneos o a la alternancia o mezcla. Como ya lo señalaba uno de los entrevistados, es muy frecuente en esta comunidad la alternancia de códigos, puesto que en las conversaciones en chedungun "el castellano se mete al tiro". No obstante, pese a que en esta comunidad prima el español, se puede percibir una motivación y un marcado optimismo hacia el aprendizaje y la preservación del chedungun.

ahí estamo' poh, tratando de volver a rescatar lo que se está perdiendo. Igual, para mí sería ... súper bueno que hablara, de hecho, lo hemos conversa'o con ... mi pareja de que ... cuando estemos nosotro' tratar de hablar lo más que podamo', para ir reforzando el tema del chedungun, conmigo y con mi hijo (Á. L., masc., Pitril).

Los sentimientos de lealtad y orgullo se instalan en ambas comunidades con un claro énfasis. Se trata de la reafirmación de una identidad que exige portar y mantener la lengua, y que ubica en un lugar de menor importancia el carácter funcional o pragmático del castellano.

Para mí sirve harto el chedungun, porque puedo entender la naturaleza con el chedungun, puedo entender el ruido del río, por ejemplo. La importancia de mi chedungun es que me enseña el conocimiento que me pasó y que me va a pasar más allá (H.M., masc., Butalelbun).

Para los hablantes de Butalelbun, la lengua es un componente trascendental para la configuración de la identidad, mientras que en Pitril, si bien se reconoce la relevancia de la lengua, se valoran además otros 
elementos identitarios tales como la participación en organizaciones y ceremonias ancestrales, vivir en la comunidad, etc.

En consecuencia, una y otra comunidad da testimonio del rol que cumplen todas las generaciones en el circuito de mantención y transmisión de la lengua. Para los hablantes, esta porta un conocimiento milenario, una sabiduría cultural, histórica, ecológica y espiritual única que se debe resguardar y que es de responsabilidad de todos.

\section{A modo de conclusión}

El proceso de incorporación e imposición del castellano, así como la conservación de la lengua originaria se expresa en un campo de heterogeneidad que se halla directamente vinculado con los procesos históricos particulares de cada territorio y también con las experiencias identitarias propias de las comunidades. Se evidencia, entonces, una diversidad en el estado de mantenimiento de la lengua en las comunidades en estudio.

En primer lugar, tanto los usos como la transmisión intergeneracional de la lengua mantienen una marcada diferencia entre ambas comunidades. Así, en Butalelbun, la lengua cumple el rol de vehículo de comunicación constante y cotidiana, con una funcionalidad preponderante y con una persistente transmisión intergeneracional. En Pitril, en cambio, el chedungun muestra más bien una carga simbólica e identitaria que funcional; es decir, es valorada como instrumento para la comunicación, pero principalmente como marcador de identidad étnica y cultural, reservorio de valores y conocimientos ancestrales. Respecto de esta visión, no solo es posible observarla en comunidades rurales o alejadas de las ciudades, sino que también en los centros urbanos (Lagos, 2011; Mellado, 2021; Rojas et al., 2016) en donde una gran parte de la población mapuche no practica el mapudungun, pero lo concibe como un elemento central para la identidad y la cultura.

Esto es coherente con los descensos en la transmisión de la lengua y la restricción de espacios comunicativos que señalaron los entrevistados. En otras palabras, aunque se observa una valoración transversalmente positiva hacia la lengua ancestral en ambas comunidades, en Pitril, se evidencia una tensión o conflicto entre el mantenimiento y uso de la lengua y su alta valoración actitudinal. Una probable hipóte- 
sis es que esta comunidad, más próxima a la Villa Ralco y a Santa Bárbara, ha estado expuesta a mayores procesos de contacto e intervención occidental (turismo, medios de comunicación y organizaciones externas) por su ubicación geográfica, lo que ha impactado en el comportamiento sociolingüístico de las familias (Henríquez y Dinamarca, 2018). La misma tensión actitudinal que evidencian los hablantes es una muestra del proceso de imposición de ideologías lingüísticas monoglósicas que relevan el rol instrumental del castellano como lengua que se asocia con mayores niveles educacionales, mayor prestigio y movilidad social; a diferencia del mapudungun que se relaciona con valores tradicionales e identitarios, pero también con el bajo progreso económico y bajo estatus social.

\section{Referencias bibliográficas}

Alonqueo, P., Wittig, F., y Huenchunao, N. (2017). Lleupeko tuwün. Un estudio exploratorio sobre niveles de competencia en mapuzungun en niños mapuches de La Araucanía. Alpha (Osorno), (44), 119-135. https://doi.org/10.4067/s071822012017000100119

Blas Arroyo, J. (2008). Sociolingüística del español. Desarrollos y perspectivas en el estudio de la lengua española en contexto social. Ediciones Cátedra.

Crystal, D. (2001). La muerte de las lenguas. Cambridge University Press.

Calvet, L. (2005). Lingüística y colonialismo. Breve tratado de la glotofagia. Fondo de Cultura Económica.

Díaz-Campos, M. (2014). Introducción a la sociolingüística hispánica. John Wiley \& Sons.

Dinamarca, J. y Henríquez, M. (2019). Una aproximación a las ideologías lingüísticas de hablantes Pewenches de la Región del Biobío. Alpha, Revista de Artes, Letras y Filosofía, 2(49), 291305. https://doi.org/10.32735/s0718-2201201900049756

Duran, T., Catriquir, D., y Hernández, A. (2007). Revitalización del mapunzungun. Una visión crítica desde la educación intercultural, la sociolingüística y la antropología. En Patrimonio cultural mapunche. Derechos lingüisticos y patrimonio cultural mapunche volumen I (pp. 107-125). Universidad Católica de Temuco. 
Edward, J. (2009). Language and identity. An introduction. Cambridge University Press.

Garrett, P. (2010). Attitudes to language. Cambridge University Press.

Ghio, E. y Fernández, M. (2008). Lingüistica sistémico funcional. Aplicaciones a la lengua española. Wadhuter Editores.

Giannelli, L. (2005). Redes y dominios de empleo de la lengua mapuche: resultados de una encuesta. En S. Dedenbach-Salazar Sáenz (Ed.), Contribuciones a las lenguas y culturas de los Andes (pp. 97-118). Shaker Verlag, BAS 42.

Godenzzi, J. C. (2007). El español de América y el español de los Andes: universalización, vernacularización y emergencia. En L. Morgenthaler García y M. Schrader-Kniffki (Eds.), La Romania en interacción: entre historia, contacto y politica: ensayos en homenaje a Klaus Zimmermann (pp. 29-50). Iberoamericana.

Godenzzi, J. C. (2017). Lenguas indoamericanas del Perú y el contacto lingüístico. Elementos para una discusión. Lenguas y Literaturas Indoamericanas, 19(1), 1-18. http://revistas.ufro.cl/ ojs/index.php/indoamericana/article/view/819

Gundermann, H., Canihuan, J., Clavería, A., y Faúndez, C. (2008). Perfil sociolingüistico de comunidades mapuches de la Región del Biobio, Araucanía, los Ríos y los Lagos. Informe de Investigación. Corporación Nacional de Desarrollo Indígena, Conadi - Universidad Tecnológica Metropolitana, UTEM.

Gundermann, H., Canihuan, J., Clavería, A., y Faúndez, C. (2009). Permanencia y desplazamiento, hipótesis acerca de la vitalidad del mapuzugun. RLA Revista de lingüística teórica y aplicada, 47(1), 37-60. https://doi.org/10.4067/s071848832009000100003

Gundermann, H., Canihuan, J., Clavería, A., y Faúndez, C. (2011). El mapuzugun, una lengua en retroceso. Atenea (Concepción), (503), 111-131. https://doi.org/10.4067/s071804622011000100006

Gundermann, H. (2014). Orgullo cultural y ambivalencia. Actitudes ante la lengua originaria en la sociedad mapuche contemporánea". Revista de Lingüística Teórica y Aplicada 52: 105132. http://dx.doi.org/10.4067/S0718-48832014000100006.

Henríquez, M. (2014). Estado del mapudungun en comunidades pewenches y lafkenches de la Región del Bío-Bío: El caso de los escolares. RLA. Revista de lingüistica teórica 
y aplicada, 52(2), 13-40. https://doi.org/10.4067/s071848832014000200002

Henríquez, M. (2015). Ámbitos de uso del mapudungun en comunidades pewenches y lafkenches de la Región del Bío-Bío. Literatura y Lingüistica, (31), 185-204. https://doi.org/10.4067/ s0716-58112015000100010

Henríquez, M. y Dinamarca, J. (2018). Actitudes lingüísticas hacia el mapudungun y el castellano: estudio exploratorio en dos comunidades pewenche del Alto Biobío. Nueva Revista del Pacífico, (69), 51-66. https://doi.org/10.4067/s071951762018000200051

Hernández-Campoy, J. (2004). El fenómeno de las actitudes y su medición en sociolingüística. Universidad de Murcia.

Hernández-Campoy, J. y Almeida, M. (2005). Metodología de la investigación sociolingüistica. Comares.

Lagos, C. (2006). Mapudungun en Santiago de Chile: vitalidad, lealtad y actitudes lingüísticas. Lenguas Modernas, (31), 97-126. https://revistas.uchile.cl/index.php/LM/article/view/45367

Lagos, C. (2011). Construcción discursiva y representaciones sociales de los mapuches urbanos en torno a su lengua nativa. Lenguas Modernas, (36), 13-32.

Lagos, C. (2012). El mapudungun en Santiago de Chile: vitalidad y representaciones sociales en los mapuches urbanos. RLA. Revista de lingüística teórica y aplicada, 50(1), 161-184. https:// doi.org/10.4067/s0718-48832012000100008

Lagos, C. (2017). Antropología lingüística y contacto lingüístico: ideologías lingüísticas en conflicto en contextos escolares de Pitril y Callaqui, Alto Bío Bío. Lenguas y Literaturas Indoamericanas, 19(1), 61-81.

Langley, A. y Royer, I. (2006). Perspectives on doing case study research in organizations. Management, 9(3), 73-86. https:// doi.org/10.3917/mana.093.0081

Mellado, I. (2021). El rol del mapudungun en la constitución de la identidad mapuche urbana: aproximación desde la antropología lingüistica [Tesis de maestría no publicada]. Universidad de Chile.

Montrul, S. (2013). El bilingüismo en el mundo hispanohablante. WileyBlackwell. 
Moreno Fernández, F. (2009). Principios de sociolingüistica y sociología del lenguaje. Ariel.

Moreno Fernández, F. (2012). Sociolingüistica cognitiva. Iberoamericana-Vervuert.

Ojeda, P. y Álvarez, J. (2014). Elementos para la construcción social del chedungun a partir del discurso en torno a la lengua de hablantes bilingües de la VIII Región. Boletín de filología, 49(2), 161-185. https://doi.org/10.4067/s071893032014000200008

Olate, A., Alonqueo, P., y Canihuán, J. (2013). Interactividad lingüística castellano/mapudungun de una comunidad rural bilingüe. Alpha (Osorno), 37, 265-284. https://doi.org/10.4067/s071822012013000200019

Olate, A., Cisternas, C., Wittig, F., y Flores, J. (2017). Los misioneros capuchinos bávaros y sus ideologías lingüísticas sobre la lengua mapuche. Nueva Revista del Pacífico 67, 130-156. https://doi.org/10.4067/S0719-51762017000200130

Olate, A. y Henríquez, M. (2010). Actitudes lingüísticas de profesores mapuche de Educación Básica: vigencia y enseñanza del mapudungun en el contexto educativo. Literatura $y$ Lingüistica, (22), 103-116. https://doi.org/10.4067/s071658112010000200008

Rojas, D. (2013). Actitudes e ideologías de hispanohablantes en torno a las lenguas indígenas en el Chile del siglo XIX. Lenguas modernas, (42), 85-98.

Rojas, D., Lagos, C., y Espinoza, M. (2016). Ideologías lingüísticas acerca del mapudungun en la urbe chilena: el saber tradicional y su aplicación a la revitalización lingüística. Chungará (Arica), 47(1), 1-11. https://doi.org/10.4067/s071773562015005000034

Thomason, S. (2001). Language contact. An introduction. Georgetown University.

Soto, E. (2014). Mapuzugun y los procesos de colonización. Universum, 29(1), 57-83.

Wittig, F. (2009). Desplazamiento y vigencia del mapudungun en Chile: un análisis desde el discurso reflexivo de los hablantes urbanos. RLA. Revista de Lingüistica teórica y aplicada, 47(2), 135-155. https://doi.org/10.4067/s071848832009000200008 
Wittig, F. y Alonqueo, P. (2018). El mapuzungun en niños mapuche de La Araucanía. Reflexiones sobre adquisición de la lengua a partir de un estudio de medición directa. Literatura y Lingüistica, (38), 213-230. https://doi.org/10.29344/0717621x.38.1634

Wittig, F. y Hernández, M. (2017). Los espacios significativos para la socialización lingüística en el discurso de jóvenes mapuche. Un acercamiento desde la investigación cualitativa. Lenguas y Literaturas Indoamericanas, 19(1), 82-100. http://revistas. ufro.cl/ojs/index.php/indoamericana/article/view/929

Zúñiga, F. (2007). Mapudunguwelaymi am? “ ¿Acaso ya no hablas mapudungun? Acerca del estado actual de la lengua mapuche". Estudios Públicos, (105), 9-24. https://doi.org/10.38178/cep. vil05.532

Zúñiga, F. y Olate, A. (2017). El estado de la lengua mapuche, diez años después. En I. Aninat, V. Figueroa, y R. González (Eds.), $E l$ pueblo mapuche en el siglo XXI (pp. 345-374). Centro de Estudios Públicos, CEP. 